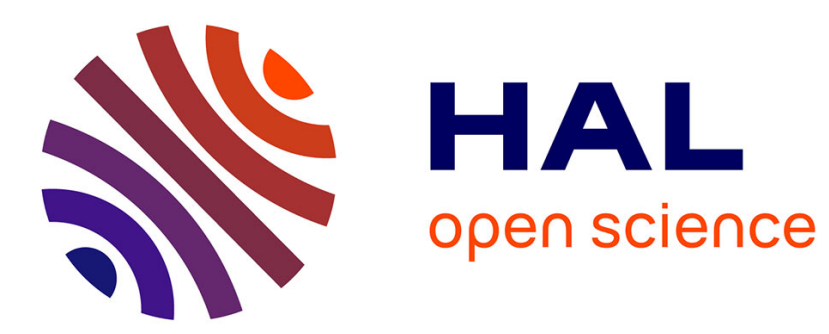

\title{
Nonlinear Diffraction of Magnetostatic Waves in Ferrite Films
}

\author{
V. Grimalsky, Yu. Rapoport, A. Slavin
}

\section{To cite this version:}

V. Grimalsky, Yu. Rapoport, A. Slavin. Nonlinear Diffraction of Magnetostatic Waves in Ferrite Films. Journal de Physique IV Proceedings, 1997, 07 (C1), pp.C1-393-C1-394. 10.1051/jp4:19971159 jpa00254804

\section{HAL Id: jpa-00254804 https://hal.science/jpa-00254804}

Submitted on 1 Jan 1997

HAL is a multi-disciplinary open access archive for the deposit and dissemination of scientific research documents, whether they are published or not. The documents may come from teaching and research institutions in France or abroad, or from public or private research centers.
L'archive ouverte pluridisciplinaire HAL, est destinée au dépôt et à la diffusion de documents scientifiques de niveau recherche, publiés ou non, émanant des établissements d'enseignement et de recherche français ou étrangers, des laboratoires publics ou privés. 


\title{
Nonlinear Diffraction of Magnetostatic Waves in Ferrite Films
}

\author{
V. Grimalsky, Yu. Rapoport and A.N. Slavin* \\ Kiev National University, 252601 Kiev, Ukraine \\ * Oakland University, Rochester, MI 48309, U.S.A.
}

\begin{abstract}
Two-dimensional nonlinear diffraction of magnetostatic waves (MSW) in ferrite films is studied numerically using the model of nonlinear parabolic equation. This equation is derived by a new method from a bilinear relation similar to the Lorentz lemma. It is shown, that in the framework of this model the evolution of two-dimensional backward volume MSW pulse leads to a collapse in the case of an infinite film, while in a film waveguide of a finite width the collapse can be avoided, and the pulse amplitude can be stabilized. Dynamics of modulation instability of both backward volume and forward volume MSW under continuous excitation is also investigated.
\end{abstract}

Magnetostatic waves (MSW) propagating in ferrite films demonstrate a wide variety of nonlinear effects, like formation of envelope solitons, modulation, decay, and kinetic instabilities, and chaotic dynamics at moderate input power levels [1]. Envelope solitons of MSW and dipole-exchange spin waves have been studied both experimentally and theoretically in various geometries, like forward volume MSW, backward volume MSW, etc. ( see [2] and Ch.9 in [1]). Theoretical interpretation of the majority of these experiments was done so far using the model of a one-dimensional nonlinear Schrödinger equation (NSE), although in real experiments the input wave packets are spatially limited in a transverse direction, and are susceptible to various transverse instabilities of nonlinear waves [3]. Experimental observation of such twodimensional instabilities have been reported recently for backward volume MSW [4].

The aim of this paper is to investigate theoretically propagation of MSW beams of finite amplitude and limited transverse width in ferrite films in a framework of a nonlinear parabolic equation model. We propose a simple new method of derivation of this model based on a bilinear relation similar to the Lorentz lemma. Detailed description of this method will be given elsewhere [5]. The bilinear relation presented below is derived from the Maxwell's equations rewritten for positive-frequency components of a nonlinear wave $\boldsymbol{E}_{l}, \boldsymbol{H}_{I}$ (index 1), and negative-frequency components of a monochromatic linear wave $\boldsymbol{E}_{2}$, $\boldsymbol{H}_{2}$ (the frequency $\boldsymbol{\omega}$ of a linear wave is the same as the carrier frequency of a nonlinear wave) :

$$
\begin{aligned}
& (c / 4 \pi) \operatorname{div}\left\{\left[\boldsymbol{E}_{2}{ }^{*} \wedge \boldsymbol{H}_{1}\right]+\left[\boldsymbol{E}_{1} \wedge \boldsymbol{H}_{2}{ }^{*}\right]\right]+(1 / 4 \pi)(\partial / \partial t)\left(\boldsymbol{H}_{1} \boldsymbol{H}_{2}{ }^{*}+\left(4 \pi \omega_{H} / \omega_{M}\right)\left(m_{l x} m_{2 x}{ }^{*}+m_{1 y} m_{2 y}{ }^{*}\right)\right\}+ \\
& +i \omega H_{2 z}{ }^{*} m_{l z}+\left(i \omega / M_{o}\right) m_{2 y}{ }^{*}\left(H_{1 y} m_{1 z}-H_{1 z} m_{l y}\right)-\left(i \omega / M_{o}\right) m_{2 x}{ }^{*}\left(H_{1 z} m_{1 x}-H_{l x} m_{l z}\right)=0 .
\end{aligned}
$$

Here $m_{x, y, z}$ are the components of the variable magnetization, $M_{O}$ is the saturation magnetization, $H_{O}$ is the external bias magnetic field directed along the $z$ axis, $\omega_{H}=\gamma H_{0}, \omega_{M}=4 \pi \gamma M_{0}$, and $\gamma$ is the gyromagnetic ratio.

Integrating relation (1) along the normal to the film plane, and substituting slowly varying amplitude $U$ for the amplitude of the wave 1 , one can obtain the evolution equation for the complex amplitude of the MSW beam $U(z, y, t)$ :

$$
\partial U / \partial t+V_{g} \partial U / \partial z+i g_{I} \partial^{2} U / \partial z^{2}+i g_{2} \partial^{2} U / \partial y^{2}+i \alpha|U|^{2} U+\Gamma U=0
$$

where $V_{g}$, is the group velocity, $g_{I}$ and $g_{2}$ are the dispersion and diffraction coefficients, $\alpha$ is the nonlinear coefficient, and $\Gamma$ is the MSW dissipation parameter. The axes $z$ and $y$ are in the film plane: $z$ is parallel to the direction of group velocity, while $y$ is perpendicular to it. The expressions for all the coefficients of Eq.(2) for all types of MSW, as well as the conditions for longitudinal (along $z$ ) and transverse (along y) instabilities of MSW are presented in Ch.9 of [1].

In this paper we used a difference method of alternating directions to study numerically the evolution of an MSW beam, governed by the two-dimensional parabolic equation (2), in the most interesting case of backward volume MSW (BVMSW) for which the conditions of modulation instability are fulfilled in both directions $\left(g_{1,2} \alpha>0\right)$. We studied both pulsed and continuous regimes of wave excitation. In the pulsed regime the general form of the input pulse was two-dimensional ( onehalf of it is shown in Fig.1). This input pulse was not only localized along the direction of propagation, but also had some variation of amplitude along the transverse direction ( $y$ axis). If the transverse variation is ignored both in the input pulse profile (Fig. 1) and in Eq. (2), the problem becomes one-dimensional, and yields a soliton solution homogeneous in $y$ direction. The profile of this soliton is shown in Fig. 2. It is well known from the analytic theory [3], that this onedimensional soliton is unstable to transverse perturbations for any sign of the diffraction coefficient $g_{2}$. Numerical results show that the evolution of the two-dimensional input pulse (Fig. 1) governed by the full parabolic equation (2) is really dramatic : in an infinite film this evolution leads to the wave collapse when the packet amplitude becomes infinite in a finite 
time. The initial stage of this two-dimensional collapse of the initial pulse is shown in Fig. 3 . The calculations were done for the following parameters: $g_{1} t_{n} / l_{z}^{2}=g_{2} t_{n} / l_{y}{ }^{2}=0.2, \alpha U_{0}^{2} t_{n}=0.5, \Gamma t_{n}=0.05$.

Parameter $t_{n}=l_{z} /\left|V_{g}\right|$ is the characteristic time scale, $l_{z}$ and $l_{y}$ are the spatial scales along the $z$ and $y$ axes, and $U_{0}$ is the maximum value of the input pulse amplitude. It is interesting (and important for practical applications) to note, that the collapse (Fig.3) can be avoided if the width of the input pulse in the $y$ direction is limited $\left(|y|<l_{y}\right)$, and boundary conditions of a "magnetic wall" type are imposed. In that case, when the input wave packet propagates in a film waveguide of a finite width, no collapse takes place and the packet amplitude is stabilized (see Fig. 4 ).

We also studied development of modulation instability (MI) of constant amplitude MSW beams having the following transverse profile: $U(z=0, y, t)=U_{0} \exp \left(-Q\left(y-y_{0}\right)^{2}\right)$. Our calculations show that the MI of forward volume MSW in our two-dimensional model takes place at higher amplitudes than in the transversely uniform case $Q=0$. It was also shown that the BVMSW beam demonstrates the effect of wave collapse at relatively low input amplitudes. At the collapse points wave amplitudes become very large, and the initial approximation of weak nonlinearity is not valid anymore.

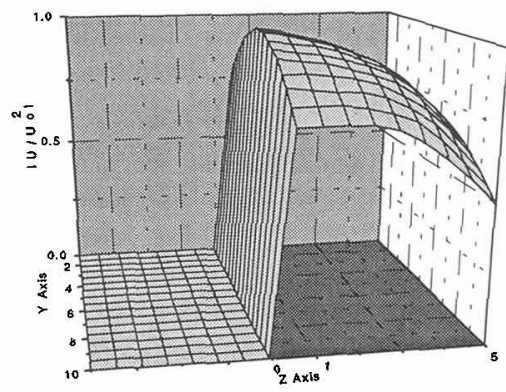

Fig.1. One half of a two-dimensional input pulse shown at the moment $t=t_{0}$.

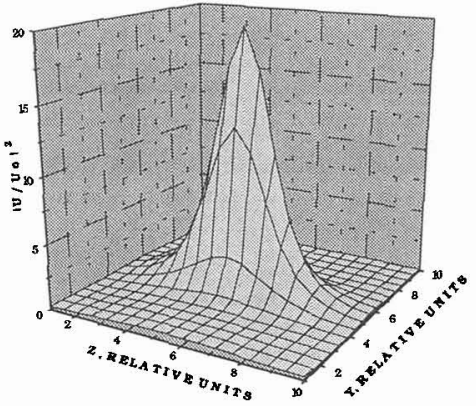

Fig. 3. Collapse of a BVMSW soiton in an infinite (in plane) film.

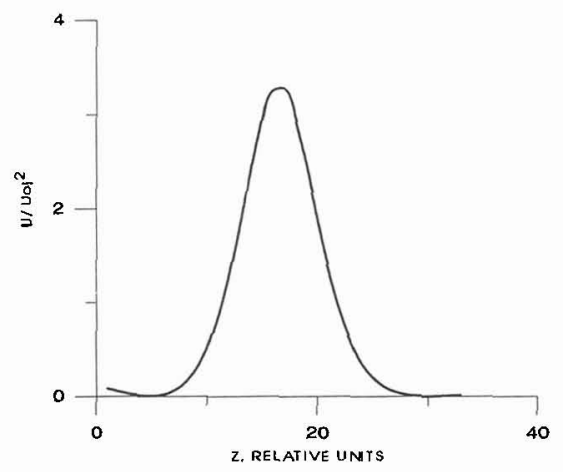

Fig. 2 One-dimensional BVMSW soliton.

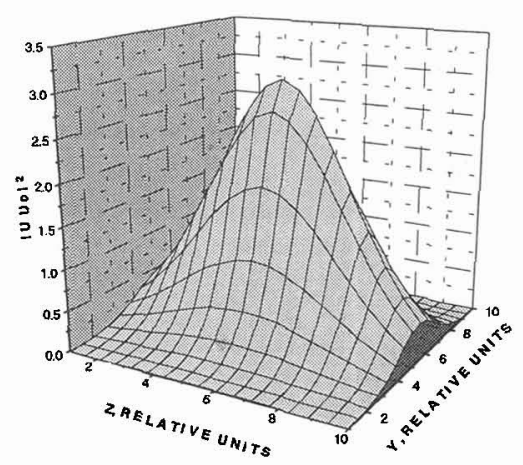

Fig.4. Stabilization of collapse in a finite-width film waveguide.

\section{References}

[1]. Wigen P.E. , ed., Nonlinear Phenomena and Chaos in Magnetic Materials (World Scientific, Singapore, 1994).

[2]. Chen M., Tsankov M.A., Nash J.M., and Patton C.E., Phys. Rev.B 49 (1994) 12773-12790.

[3]. L'vov V.S., Wave Turbulence under Parametric Excitation (Springer, Berlin, 1994) pp. 27-34 .

[4]. Boyle J.W. et al. , Phys. Rev.B 53 (1996) 12173-12181.

[5]. Grimalsky V.V., Rapoport Y. G., and Slavin A.N., Phys. Rev. B (1997), to be published . 